\title{
Examining the distribution and dynamics of impervious surface in different function zones in Beijing
}

\author{
QIAO Kun ${ }^{1,2,3}$, ZHU Wenquan ${ }^{1,2}$, "HU Deyong ${ }^{3}$, HAO Ming ${ }^{4}$, \\ CHEN Shanshan ${ }^{3}$, CAO Shisong ${ }^{3}$
}

1. State Key Laboratory of Earth Surface Processes and Resource Ecology, Beijing Normal University, Beijing 100875, China;

2. Institute of Remote Sensing Science and Engineering, Faculty of Geographical Science, Beijing Normal University, Beijing 100875, China;

3. College of Resource Environment and Tourism, Capital Normal University, Beijing 100048, China;

4. Nuclear Industry of China Geotechnical Engineering Co. Ltd, Shijiazhuang 050021, China

\begin{abstract}
Impervious surface (IS) is often recognized as the indicator of urban environmental changes. Numerous research efforts have been devoted to studying its spatio-temporal dynamics and ecological effects, especially for the IS in Beijing metropolitan region. However, most previous studies primarily considered the Beijing metropolitan region as a whole without considering the differences and heterogeneity among the function zones. In this study, the subpixel impervious surface results in Beijing within a time series (1991, 2001, 2005, 2011 and 2015) were extracted by means of the classification and regression tree (CART) model combined with change detection models. Then based on the method of standard deviation ellipse, Lorenz curve, contribution index $(\mathrm{Cl})$ and landscape metrics, the spatio-temporal dynamics and variations of IS (1991, 2001, 2011 and 2015) in different function zones and districts were analyzed. It is found that the total area of impervious surface in Beijing increased dramatically during the study period, increasing about $144.18 \%$. The deflection angle of major axis of standard deviation ellipse decreased from $47.15^{\circ}$ to $38.82^{\circ}$, indicating the major development axis in Beijing gradually moved from northeast-southwest to north-south. Moreover, the heterogeneity of impervious surface's distribution among 16 districts weakened gradually, but the $\mathrm{Cl}$ values and landscape metrics in four function zones differed greatly. The urban function extended zone (UFEZ), the main source of the growth of IS in Beijing, had the highest $\mathrm{Cl}$ values. Its lowest $\mathrm{Cl}$ value was 1.79 that is still much higher than the highest $\mathrm{Cl}$ value in other function zones. The core function zone (CFZ), the traditional aggregation zone of impervious surface, had the highest contagion index (CONTAG) values, but it contributed less than UFEZ due to its small area. The $\mathrm{Cl}$ value of the new urban developed zone (NUDZ) increased rapidly, and it increased from negative to positive and multiplied, becoming an
\end{abstract}

Received: 2017-09-20 Accepted: 2017-10-30

Foundation: The National Basic Research Program of China, No.2015CB953603; National Natural Science Foundation of China, No.41671339; State Key Laboratory of Earth Surface Processes and Resource Ecology, No.2017FX-01(1)

Author: Qiao Kun (1989-), PhD Candidate, specialized in remote sensing of resource and environment, remote sensing of vegetation. E-mail: qiaoyingying2009@126.com

"Corresponding author: Hu Deyong (1974-), PhD and Professor, specialized in remote sensing of resource and environment, remote sensing monitoring of natural disasters. E-mail: deyonghu@163.com 
important contributor to the rise of urban impervious surface. However, the ecological conservation zone (ECZ) had a constant negative contribution all the time, and its $\mathrm{Cl}$ value decreased gradually. Moreover, the landscape metrics and centroids of impervious surface in different density classes differed greatly. The high-density impervious surface had a more compact configuration and a greater impact on the eco-environment.

Keywords: impervious surface; landscape metrics; classification and regression tree (CART); function zones; Lorenz curve; contribution index

\section{Introduction}

In 2014, the Chinese government released the National New-Type Urbanization Plan, which notes that there have been 142 cities with a population more than one million and 10 cities with a population of more than ten million. In addition, China's urbanization rate is predicted to reach $60 \%$ by 2020 (Xinhua News Agency, 2014). The human activities in urban area are the most intense, especially in metropolitan regions. With the rapid acceleration of urban development, large numbers of natural surfaces have been replaced by anthropogenic surfaces, such as asphalt roads and high buildings, which inevitably have resulted in various effects on the eco-environment (Peng, 2008; Sun, 2013; Peng et al., 2016b). The dynamics of urban underlying surface and its effects on eco-environment have received more and more attention from the governments and researchers.

The impervious surface is usually defined as the collection of anthropogenic landforms that water cannot directly infiltrate into, including rooftops, roads and parking lots (Chester et al., 1996; Qiu et al., 2011). The dynamics of impervious surface impact urban regional climate by altering the thermal environment and water quality (Brun and Band, 2000; Gillies et al., 2003; Xie et al., 2009; Kuang et al., 2011; Fu and Weng, 2016), due to its characteristics of strong capacity of heat storage and weak ability of evapotranspiration, which impede the transmission of airflow (Nie, 2013). Impervious Surface Percentage (ISP) is the percentage of impervious surface area in a unit surface area (Gao et al., 2010; Zhang et al., 2010; Song, 2014). It is a kind of continuous spatial data, which can be used to describe the urban land use pattern comprehensively and accurately, revealing the urban landscape dynamics and change processes quantitatively. In addition, ISP is not susceptible to the change of season, phenology and other external influences. Therefore, it has been recognized as a key indicator in environmental assessment.

As China's political, cultural center and international exchange center, as well as the science and technology innovation center, Beijing has experienced a rapid urbanization process in recent years, and has become one of the cities with the highest urbanization rate in northern China. Currently, numerous research efforts have been devoted to quantifying the spatio-temporal dynamics of impervious surface in Beijing and its ecological influences (Peng et al., 2016a). Xiao et al. (2007) and Wang et al. (2014) adopted CART (Classification and Regression Tree) and V-I-S (Vegetation-Impervious surface-Soil) model, respectively, to retrieve the distribution of impervious surface in Beijing urban core area, and found that the impervious surface areas increased rapidly each year. Moreover, the ratio of impervious surface decreased from the urban core to the suburbs gradually, presenting a strong spatial gradient. A long time-series spatial data of impervious surface and land surface temperature (LST) in Beijing urban core area were retrieved from Landsat series data and land cover data 
by Cui et al. (2015) and Hao et al. (2015), respectively. It is reported that the impervious surface in Beijing mainly extended along the ring roads. Besides, it is also found that there was a close relationship between the ISP and LST, and the negative impact of impervious surface on urban thermal environment was greater than the positive impact of vegetation. However, most previous studies primarily considered Beijing metropolitan region as a whole and focused on the impervious surface in major zones without considering the differences and heterogeneity of impervious surface's distribution among the function zones. As a result, the development pattern and change process of impervious surface in Beijing cannot be interpreted fully.

In addition, there are various shape characteristics and spatial arrangement features of impervious surface in Beijing's function zones, which are important for quantifying urban sprawl and environment. These different characteristics and features can lead to the heterogeneity of impervious surface's distribution (Zhou et al., 2011; Liu and Yang, 2015). Therefore, it is necessary to quantify the landscape pattern of impervious surface accurately to expound the dynamics of urban expansion. The integration of landscape ecology theory and remote sensing technology can be quite useful for analyzing the spatial patterns and ecological impacts of impervious surface at different scales. However, previous landscape studies about the impervious surface are mostly based on the "hard" classification results, few researchers focused on the "soft" classification results, the subpixel impervious surface (Peng et al., 2010; Zhang et al., 2015). The "soft" classification results of impervious surface provide more realistic descriptions and information of urban landscape structure than the "hard" classification results.

The subpixel impervious surface results in Beijing metropolitan region within a time series were retrieved from the CART and change detection models, and then the distribution and spatio-temporal dynamics of impervious surface were analyzed. To clarify the role and status of different function zones in Beijing, the spatio-temporal dynamics of impervious surface in each function zone and district were discussed. Meanwhile, the contribution index of each function zone was calculated. Through converting continuous ISP data to discrete data of ISP density classes, the landscape metrics of impervious surface in each function zone were further calculated, and then the landscape pattern of impervious surface was analyzed, which aims to provide relatively reliable decision bases for urban planning and management.

\section{Study area and data}

\subsection{Study area}

Beijing is situated in North China between $115.7^{\circ}-117.4^{\circ} \mathrm{E}$ and $39.4^{\circ}-41.6^{\circ} \mathrm{N}$. The northwest of the city with a higher terrain is surrounded by low mountains and hills, and the southeast side with a flat terrain slopes to the Bohai Rim (Figure 1). It is characterized by a typical continental monsoon climate with distinct seasons. Beijing has an area of $16412 \mathrm{~km}^{2}$, of which, the area of major zones is $1369 \mathrm{~km}^{2}$. The population and urbanization rate of Beijing increased rapidly in the past decades. The urbanization rate and the resident population have reached $86.5 \%$ and 21.705 million by 2015 , respectively. The urban population has increased to 18.777 million. Overall, the process of urbanization in Beijing is accelerating. 


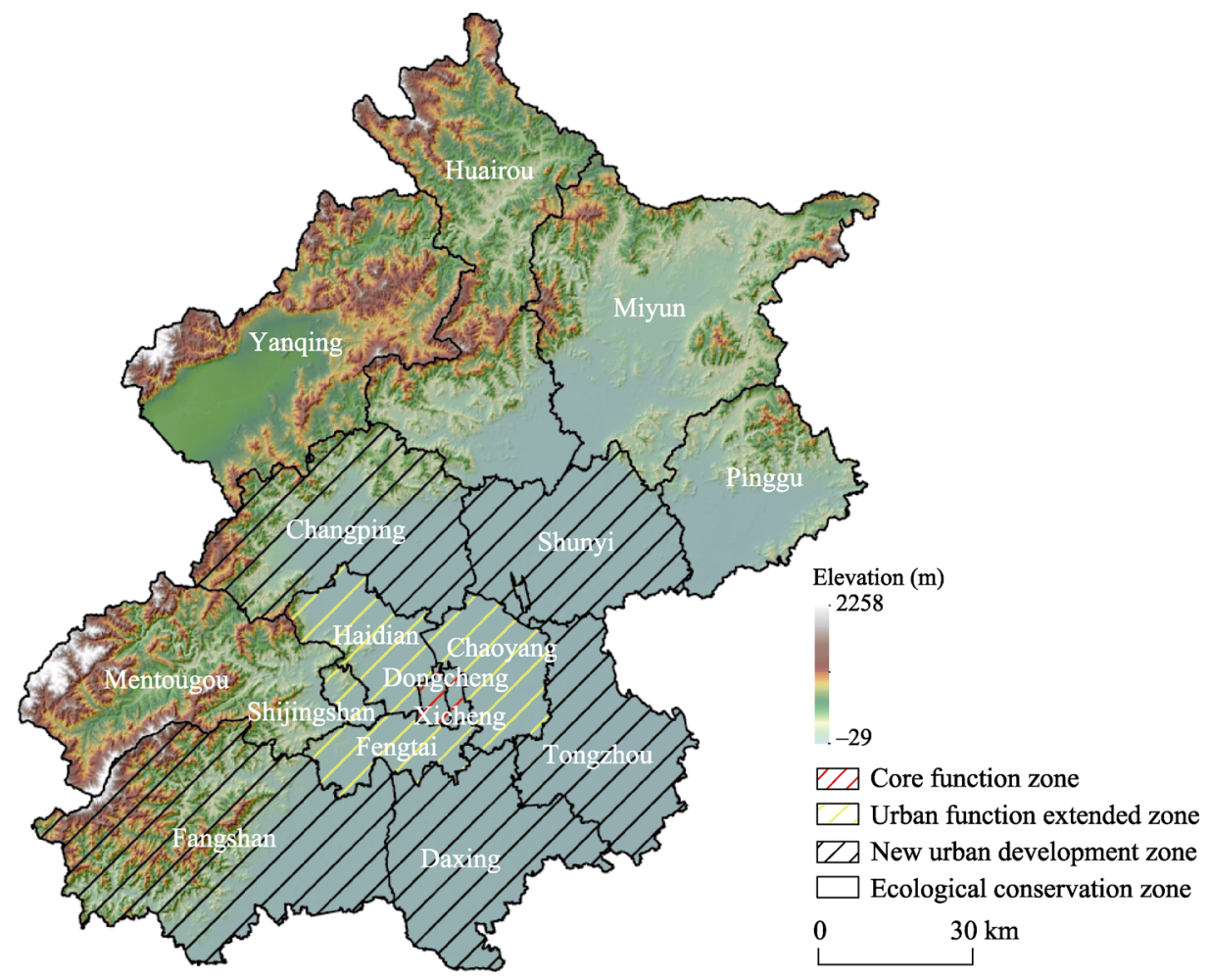

Figure 1 Location of the study area and its function zones

According to the government documents and The Master Plan For Beijing City (2004-2020) released in 2005, the 16 districts of Beijing have been divided into four function zones (Figure 1), including: (1) core function zone (CFZ), which is the center of Beijing, mainly characterized by the political, cultural and international exchange functions; (2) urban function extended zone (UFEZ), which is the important industrial cluster for economy, education, modern services and tourism; (3) new urban development zone (NUDZ), which is the agglomeration of high technology, modern manufacturing and agricultural industries, and is also a major driver for Beijing's future development; and (4) ecological conservation zone (ECZ), which plays a significant role in protecting water resource and environment, and serves as an ecological barrier for Beijing.

\subsection{Data}

Landsat 5 thematic mapping (TM) data acquired in 1991 (May 16), 2001 (May 19 and August 31), 2005 (May 6 and November 14) and 2011 (June 8), together with Landsat 8 operational land imager (OLI) data acquired in 2015 (February 11 and September 7) were selected as the main data source to retrieve ISP. All the above data are with a spatial resolution of 30 $\mathrm{m}$. Moreover, a QuickBird image covering the major zones was used to collect the training/reference data for obtaining and assessing the mapping results of ISP in 2005. The QuickBird image with a high resolution of $2.4 \mathrm{~m}$ was obtained on $5 \mathrm{July}, 2005$. The DMSP/OLS and VIIRS/NPP data in 1992, 2001, 2005, 2011 and 2016 were collected simultaneously as auxiliary data for the simulation of impervious surface. All the data were geometrically corrected and matched, and re-projected into the UTM/WGS 84 projection with a 
pixel size of $30 \mathrm{~m}$ by $30 \mathrm{~m}$ for all bands.

\section{Methods}

\subsection{Retrieval of impervious surface}

CART model is one of the commonly used methods for ISP retrieval. It is a decision tree model conducting a binary recursive partitioning process, which can deal with mass, nonlinear and high-dimensional data effectively. Furthermore, it allows both continuous and discrete variables to be put into CART model, which is conducive to ISP retrieval at large scales. Currently, impervious surface data retrieved by CART model has been added to the US National Land Cover Database (Yang et al., 2003). Thus, the CART model was used to obtain ISP data in Beijing in this study, and the process consists of several steps: (1) The binary classification result of impervious surface was obtained from the QuickBird image by means of unsupervised classification and recode, and then the training and reference data of ISP over $30 \mathrm{~m} \times 30 \mathrm{~m}$ grids were derived from the classification result by means of neighborhood block statistics; (2) the simulation model was built by using 7 spectral bands of Landsat $5 \mathrm{TM}, \mathrm{DMSP} / \mathrm{OLS}$ and VIIRS/NPP night light data as independent variables, the ISP data derived from the QuickBird image as target variable, and then the mapping result of ISP in 2005 was retrieved from the simulation model; and (3) based on the CART simulation model and change detection models, the mapping results of ISP in 1991, 2001, 2011 and 2015 were derived from the Landsat data combined with ISP results in 2005. It should be noted that we assumed the ISP always increases with urban sprawl in the modeling process, since most urbanization processes are irreversible. Therefore, based on ISP result in 2005 and night light data in each year, the ISP data in high urbanization area were collected as the sample data for other years.

The simulation model was evaluated by 10 -fold cross-validation method. It means the training data set was divided into 10 blocks of roughly equal size. For each block in turn, a model is built from the data in the remaining blocks and tested using the holdout block (Yang et al., 2003). The ISP result in 2005 was evaluated by the reference data derived from the QuickBird image. Meanwhile, the ISP results in other years were assessed by the ISP data in high urbanization area. The ISP data in high urbanization area were derived based on ISP data in 2005 and night light data in each year (note the assumption that the ISP values of high urbanization area changed little during 1991-2015). Besides, the high resolution images of Google Earth were used as supplementary data for accuracy evaluation.

\subsection{Standard deviation ellipse and Lorenz curve}

Standard deviation ellipse analysis is one of the classical methods for analyzing directional features of spatial distribution. The spatial pattern and dominant direction of spatial elements can be measured well by the ellipse. To be specific, the aggregation of elements can be described by the elliptic semi-axial length. The deflection angle of ellipse from the north is a measurement of spatial predominant direction ( $\mathrm{Li}$ et al., 2015). In order to determine the spatial dynamics of ISP in Beijing, the standard deviation ellipses of ISP results were calculated. The formulas for the parameters in an ellipse are presented in the studies of David 
(1999) and Lauren et al. (2010).

The Lorenz curve was proposed by M. Lorenz for describing the inequality in distribution of wealth, land and income among social classes or other population units. The frequency accumulation is commonly used to depict the inequality, and the equality or inequality in distribution of income can be demonstrated intuitively by Lorenz curve (Zhang et al., 2007). In addition, the spatial density and heterogeneity of geographical elements can also be depicted vividly by Lorenz curve (Yang et al., 2012). Therefore, the Lorenz curve was used to analyze the spatial heterogeneity of ISP.

\subsection{Contribution index}

In order to quantify the roles of each function zone in Beijing, the contribution index of each zone was calculated using Eq. (1)

$$
C I=\left(\overline{I S P_{F}}-\overline{I S P}\right) \times\left(S_{F} / S\right)
$$

where $C I$ denotes the contribution index; $\overline{I S P_{F}}$ and $\overline{I S P}$ denote the average ISP values of each function zone and Beijing, respectively; and $S_{F}$ and $S$ denote the total area of each function zone and Beijing, respectively.

\subsection{Landscape metrics}

To analyze the spatial characteristics and dynamics of impervious surface landscape, the weighted centroid and landscape metrics of impervious surface were calculated. The weighted centroid was used to measure the dynamics of impervious surface in different density classes, while landscape metrics were used to analyze the shape characteristics and spatial arrangement features of impervious surface.

The contagion index (CONTAG) of landscape level was selected to analyze the aggregation of impervious surface landscape in Beijing metropolitan region. Higher CONTAG values indicate a more connected landscape, while lower values indicate less connected. The patch density (PD) and landscape shape index (LSI) were selected to describe the spatial fragmentation and shape complexity of impervious surface in different density classes. The higher the PD and LSI values are, the more the fragmented and complex is the landscape (Huang et al., 2012).

The impervious surface results in Beijing were divided into 5 density classes with an equal interval. That is: low-density impervious surface $(0<\mathrm{ISP} \leqslant 0.2)$, medium low-density impervious surface $(0.2<\mathrm{ISP} \leqslant 0.4)$, medium-density impervious surface $(0.4<\mathrm{ISP} \leqslant 0.6)$, medium high-density impervious surface $(0.6<\mathrm{ISP} \leqslant 0.8)$ and high-density impervious surface $(0.8<\mathrm{ISP} \leqslant 1.0)$.

\section{Results}

\subsection{The spatial dynamics of impervious surface in Beijing}

Evaluated by the ISP result derived from the high resolution image (i.e., QuickBird) in 2005, the simulation model of 2005 has a good performance, and the average error (AE), relative error (RE) and correlation coefficient (R) of ISP results are $12.8 \%, 0.39$ and 0.86 , respec- 
tively. The accuracies of simulated ISP results in other years (1991, 2001, 2011 and 2015) are shown in Table 1.

Table 1 Accuracies of simulated ISP results in different years

It can be seen that the $\mathrm{AE}$ value in each year was less than $14.5 \%$, RE was less than 0.44 , and $\mathrm{R}$ was larger than 0.73 .

The impervious surface in Beijing developed rapidly during 1991-2015

\begin{tabular}{cccc}
\hline Year & $\begin{array}{c}\text { Average } \\
\text { error (\%) }\end{array}$ & $\begin{array}{c}\text { Relative } \\
\text { error }\end{array}$ & $\begin{array}{c}\text { Correlation } \\
\text { coefficient }\end{array}$ \\
\hline 1991 & 10.40 & 0.43 & 0.73 \\
2001 & 8.90 & 0.36 & 0.80 \\
2011 & 8.60 & 0.44 & 0.76 \\
2015 & 14.50 & 0.41 & 0.78 \\
\hline
\end{tabular}

(Figure 2). The spatial pattern of impervious surface developed as a single core mode. From 1991 to 2015, there was a clear expansion of impervious surface area, spreading from CFZ to NUDZ and continuously expanding. The NUDZ became a new aggregation center of impervious surface in 2015 , and the ISP values also increased dramatically.
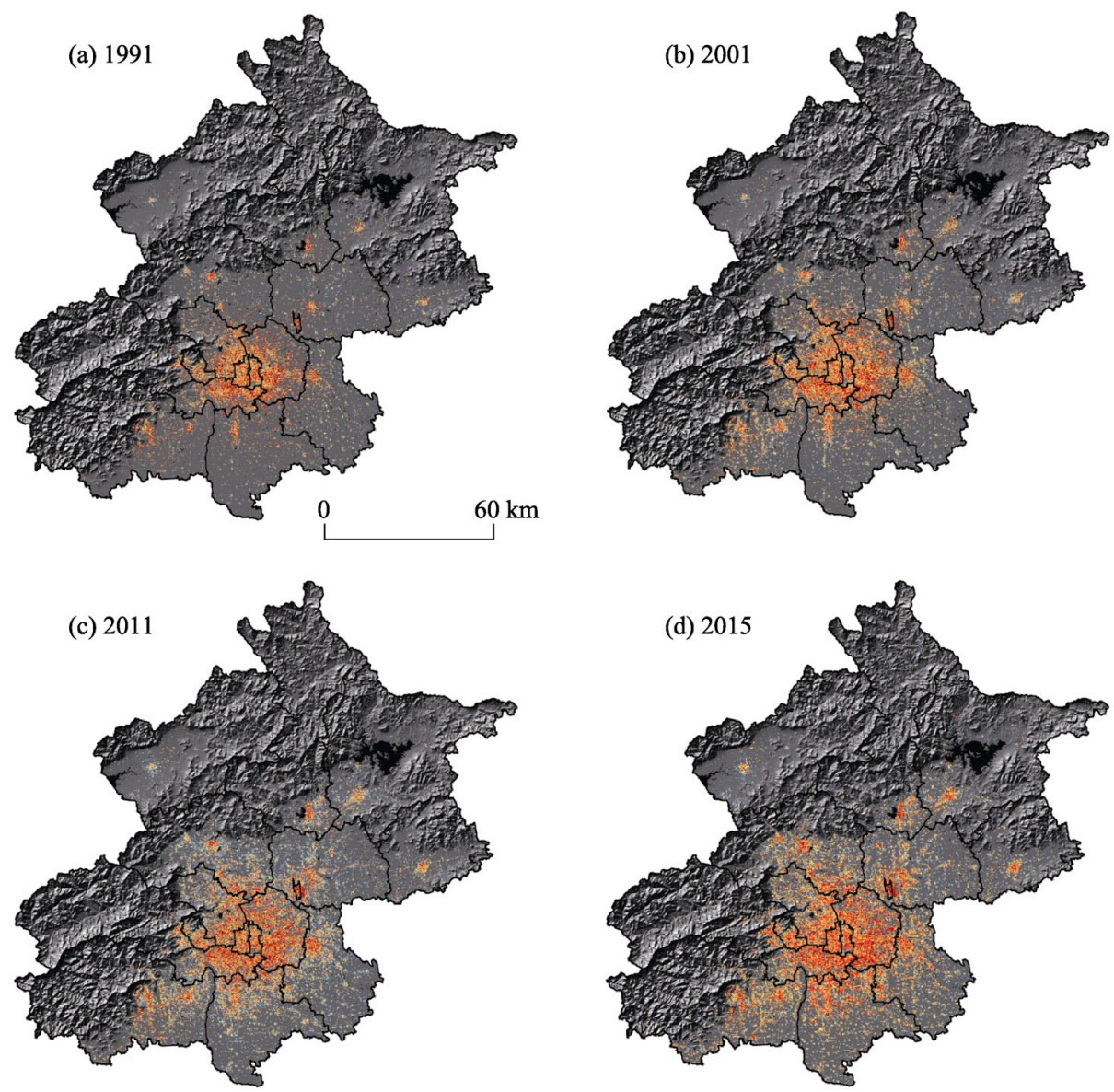

Impervious surface percentage (\%)

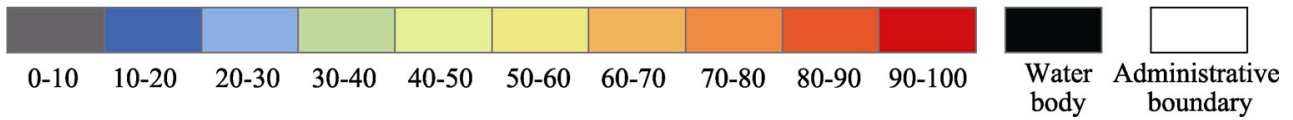

Figure 2 The spatial pattern of impervious surface percentage in Beijing in different years 
The total absolute values of area of impervious surface in Beijing in 1991, 2001, 2011 and 2015 were $714.69 \mathrm{~km}^{2}, 1109.65 \mathrm{~km}^{2}$, $1429.77 \mathrm{~km}^{2}$ and $1745.13 \mathrm{~km}^{2}$, respectively, increasing by about $144.18 \%$ from 1991 to 2015. At the same time, the average values of ISP increased significantly, and Beijing experienced a rapid urbanization process during 1991-2015.

The size of standard deviation ellipse was getting smaller from the year 1991 to 2015, and meanwhile, the ellipse moved northward gradually (Figure 3). The deflection angle of major axis decreased from $47.15^{\circ}$ in 1991 to $38.82^{\circ}$ in 2015 (Table 2), indicating there was a tendency that the major development axis in Beijing moved from northeast-southwest to north-south gradually. However, northeast-southwest profile was still the main direction of urban growth.

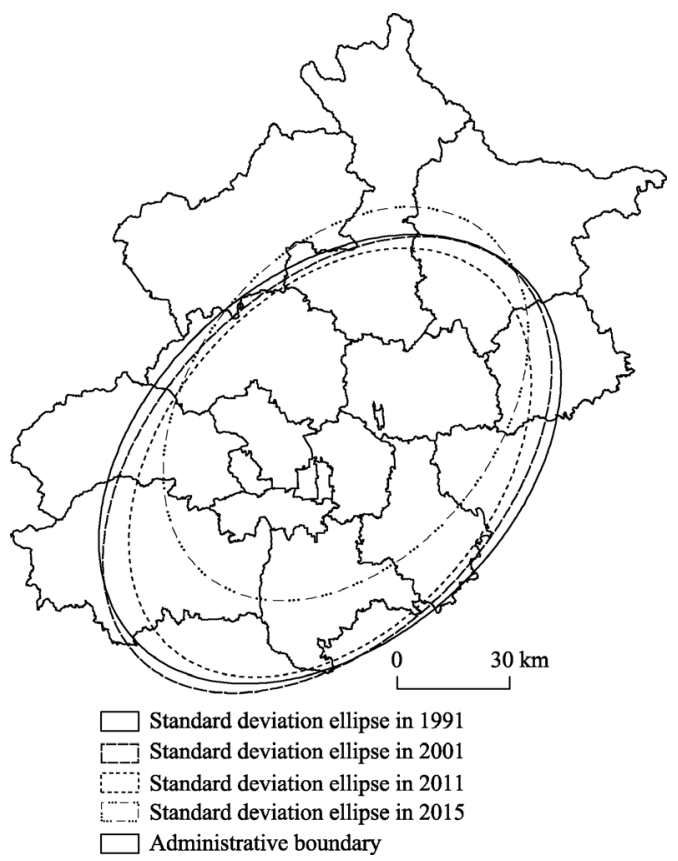

Figure 3 The spatial pattern evolution of impervious surface in Beijing from 1991 to 2015

The semi-axial length of standard deviation ellipse also changed a lot from 1991 to 2015 (Figure 2 and Table 2). During 1991-2001, the semi-major axial length increased from 72.37 $\mathrm{km}$ to $73.34 \mathrm{~km}$, and the semi-minor axial length decreased from $49.42 \mathrm{~km}$ to $46.88 \mathrm{~km}$, indicating the impervious surface's distribution showed a weak discretization tendency in the major axis and an aggregation tendency in the minor axis. During 2001-2015, the semi-major axial length decreased from $73.34 \mathrm{~km}$ to $60.13 \mathrm{~km}$, and the semi-minor axial length continued to decrease from $46.88 \mathrm{~km}$ to $41.41 \mathrm{~km}$, which demonstrates that both the impervious surface in major axis and minor axis showed an aggregation tendency.

Table 2 The parameter values of standard deviation ellipses in different years

\begin{tabular}{cccc}
\hline Year & Deflection angle $\left(^{\circ}\right)$ & Semi-major axial length $(\mathrm{km})$ & Semi-minor axial length $(\mathrm{km})$ \\
\hline 1991 & 47.15 & 72.37 & 49.42 \\
2001 & 43.79 & 73.34 & 46.88 \\
2011 & 40.05 & 66.37 & 44.69 \\
2015 & 38.82 & 60.13 & 41.41 \\
\hline
\end{tabular}

\subsection{The spatial dynamics of impervious surface in different function zones}

The spatial Lorenz curve of impervious surface was getting closer to the standard line from 1991 to 2015 (Figure 4), indicating the distribution of impervious surface among Beijing's 16 districts became equal gradually. The UFEZ had the highest CI values and contributed the most to the urban impervious surface (Figure 5). Its average ISP values were much higher than those in Beijing. Besides, the CI value increased continually from 1991 to 2015. The CFZ and NUDZ were important contributors to the rise of urban impervious surface. The average ISP values of CFZ were the highest. However, CFZ contributed less than UFEZ 
owing to its small area, and its CI values were stable and relatively low. From 1991 to 2015 , the CI value of NUDZ increased from negative to positive and multiplied. The role of CFZ was replaced by NUDZ gradually. The ECZ had a constant negative contribution, and its CI value decreased continually. It demonstrates that although the total area of impervious surface in ECZ increased, the average ISP values in this zone were much less than those in Beijing.

The CI values of different districts and function zones differed greatly. Chaoyang district located in UFEZ had the highest CI value of 1.21 in 2015, and Pinggu district located in ECZ had the lowest CI value of -1.15 in 2015. In addition, the CI values of different districts in the same function zones also differed greatly, except for the two districts in CFZ. Yet the variation trend of CI

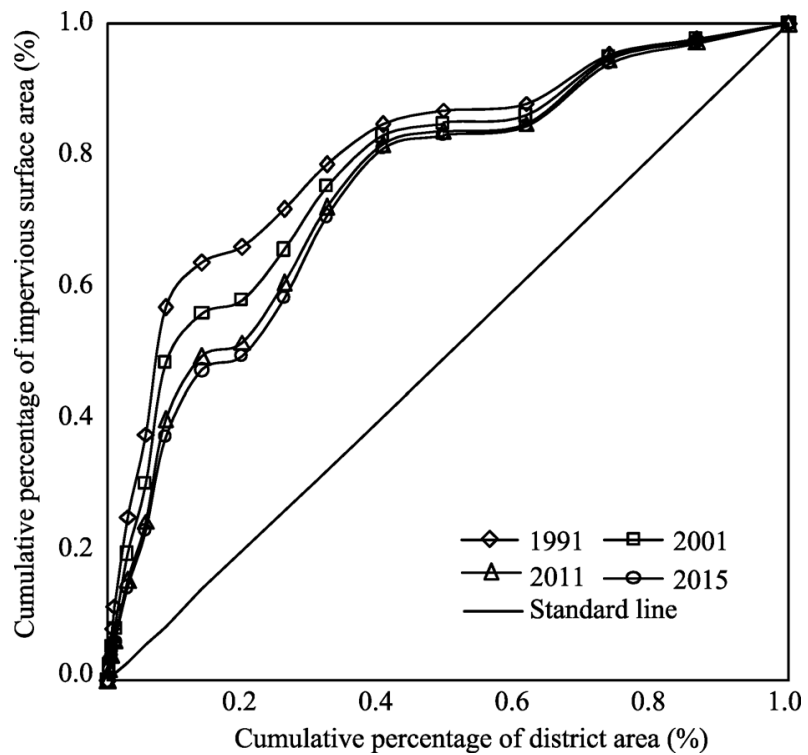

Figure 4 The spatial Lorenz curve in Beijing metropolitan region in 1991, 2001, 2011 and 2015

The horizontal axis represents the cumulative percentage of area of 16 districts in Beijing, and the vertical axis represents the cumulative percentage of the impervious surface proportion in each district. The straight line in the middle is the "standard line". The closer the spatial Lorenz curve is to the standard line, the more equally distributed is the impervious surface. On the contrary, a departure from the standard line represents an inequality in the impervious surface's distribution. values in districts was almost consistent with that in function zones.

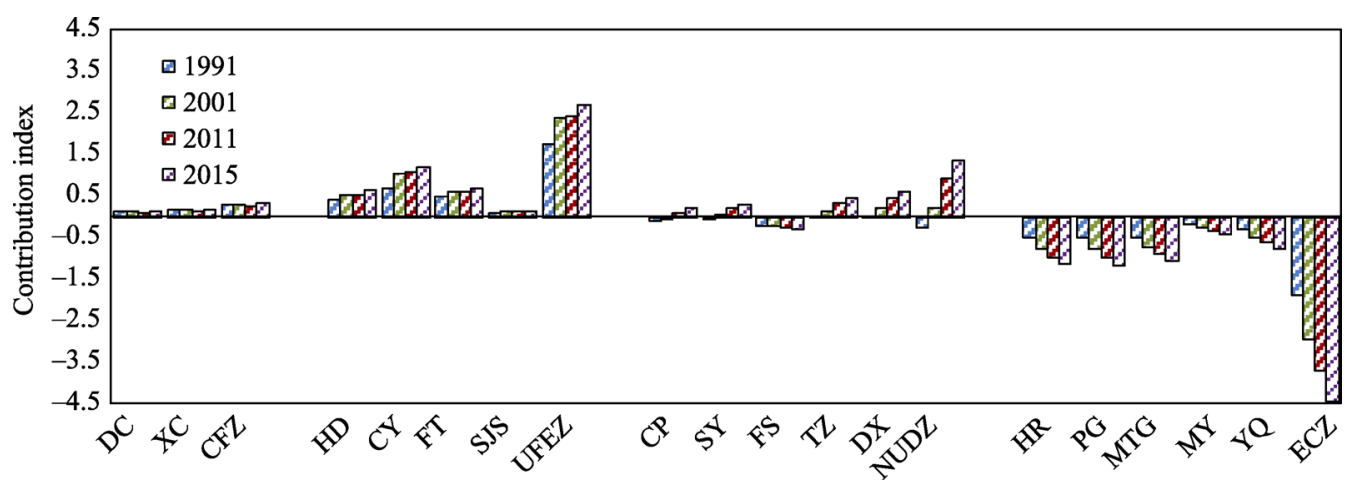

Figure 5 The contribution of each function zone and district to the growth of impervious surface in Beijing. The horizontal axis represents the 16 districts and 4 function zones. DC represents Dongcheng district, XC represents Xicheng district, HD represents Haidian district, CY represents Chaoyang district, FT represents Fengtai district, SJS represents Shijingshan district, CP represents Changping district, SY represents Shunyi district, FS represents Fangshan district, TZ represents Tongzhou district, DX represents Daxing district, HR represents Huairou district, PG represents Pinggu district, MTG represents Mentougou district, MY represents Miyun district, and YQ represents Yanqing district.

\subsection{The landscape pattern dynamics of impervious surface in function zones}

\subsubsection{The variations of centroid}

CFZ is the traditional aggregation zone of impervious surface in Beijing (Figure 6). The centroids of impervious surface in different density classes were mainly located in the area 
between Xicheng district in CFZ and Chaoyang district in UFEZ during the study period. From 1991 to 2015, the landscape patches of high-density and medium-density impervious surface developed fast in the northeast at early stage, and then developed rapidly in the northern part, so the centroid tended to move to northeast at first and then to north. The centroid of medium-high density impervious surface constantly moved to the northeast during 1991 to 2015. For the medium-low density impervious surface, its centroid moved to the southwest during 1991 to 2001, then moved to the northeast during 2001 to 2011, and finally moved to the northwest. The centroid migration trajectory of low-density impervious surface showed a U-shaped pattern, and the centroid first moved to the south, then to the west and finally to the north. Furthermore, it is found that the centroids' movements during 2011 to 2015 were much larger than those during other periods, indicating there was an obviously directional trend in urban development during this period. On the contrary, the centroids' movements were the smallest during 2001 to 2011, thus, the urban area developed along multi-directions.

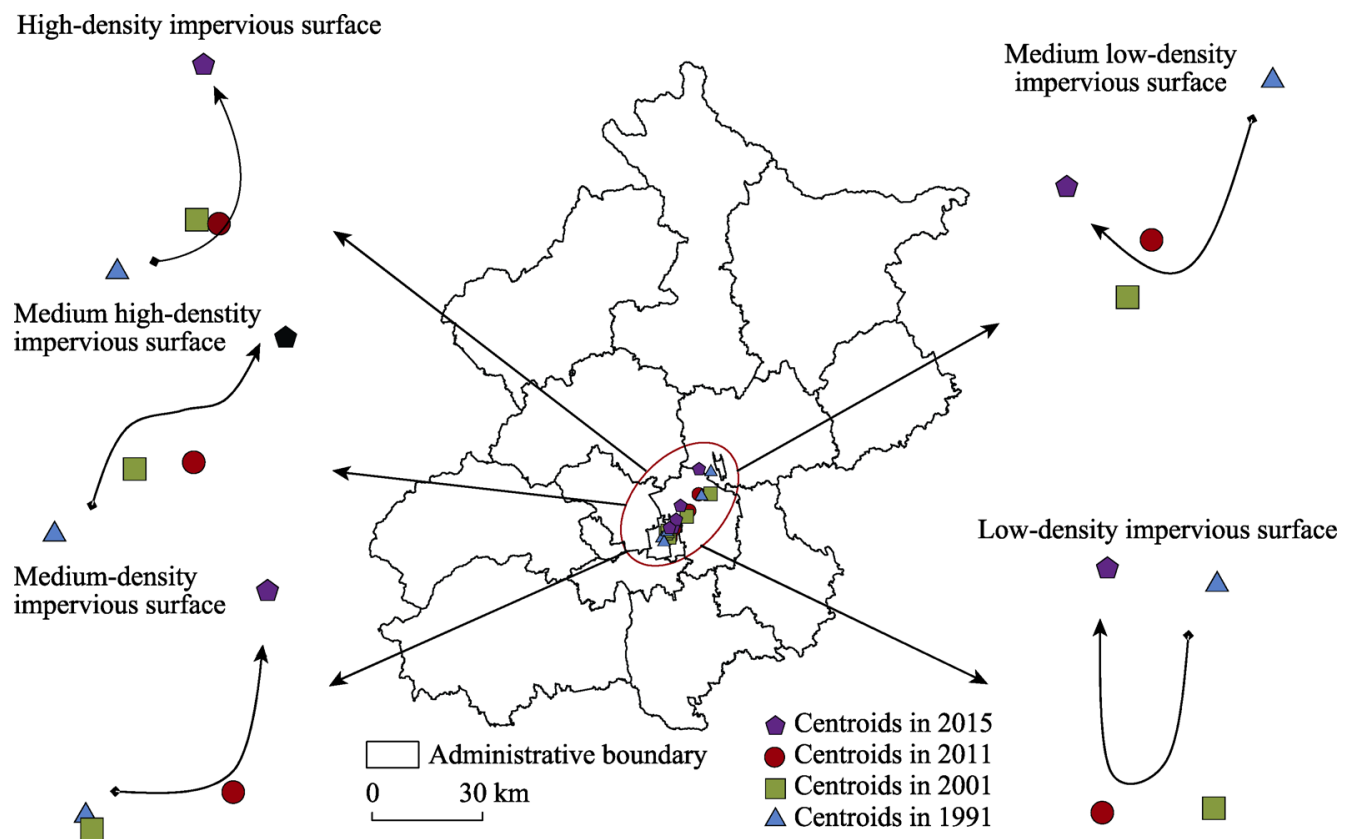

Figure 6 The migration of spatial centroids of impervious surface landscape in Beijing

\subsubsection{The landscape metrics of impervious surface}

The CONTAG values in CFZ were the highest in the four function zones in all the four years (Table 3). It means the impervious surface landscape in CFZ was more connected and compact than that in other zones. Besides, the landscape patches of impervious surface in both NUDZ and ECZ developed fast. As a result, the gap of CONTAG values between these two zones and UFEZ was narrowed. From 1991 to 2015, the CONTAG values in CFZ and UFEZ decreased at first and then increased. However, unlike CFZ and UFEZ, the CONTAG values in NUDZ and ECZ increased all the time, indicating that the spatial aggregation of impervious surface increased over time. The CONTAG values exhibited a slight variation in Beijing 
metropolitan region. In the whole urbanization process of Beijing, increasing the patches of impervious surface outwards was the main way of urban expansion in the early-developed stage. However, during the later developed period, the main way of urban expansion had changed to enhancing the connectedness among patches.
Table 3 The CONTAG values of each function zone and Beijing in different years

\begin{tabular}{cccccc}
\hline Year & CFZ & UFEZ & NUDZ & ECZ & Beijing \\
\hline 1991 & 31.75 & 23.20 & 9.04 & 10.62 & 13.19 \\
2001 & 31.22 & 23.41 & 11.05 & 10.52 & 13.42 \\
2011 & 30.64 & 19.50 & 12.35 & 12.35 & 12.61 \\
2015 & 36.90 & 24.17 & 16.03 & 19.96 & 15.72 \\
\hline
\end{tabular}

The values of LSI and PD in both CFZ and UFEZ increased with the rise of ISP and then decreased (Figures 7 and 8 ). This means as the ISP rose, the landscape structure and shape of impervious surface became increasingly fragmented and irregular at first, and then became less fragmented and less complex. In NUDZ and ECZ, the values of LSI and PD showed a downtrend, indicating the fragmentation and complexity of impervious surface landscape attenuated as the ISP rose. Besides, it is noticeable that in all function zones, the landscape structure and shape of high-density impervious surface were much more compact and aggregative than other types of impervious surface (note the landscape metrics of low-density impervious surface were the lowest in CFZ due to its small area). The changing trend of LSI and PD in Beijing metropolitan region was similar to that in NUDZ.
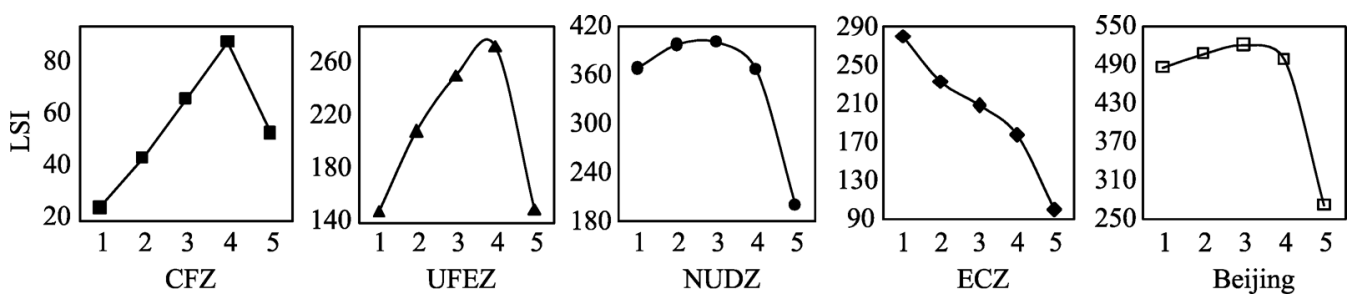

Figure 7 The LSI values of different impervious surface's types in four function zones and Beijing in 2015 The number 1, 2, 3, 4 and 5 in the x-axis represent the low-density impervious surface, medium low-density impervious surface, medium-density impervious surface, medium high-density impervious surface and high-density impervious surface, respectively.
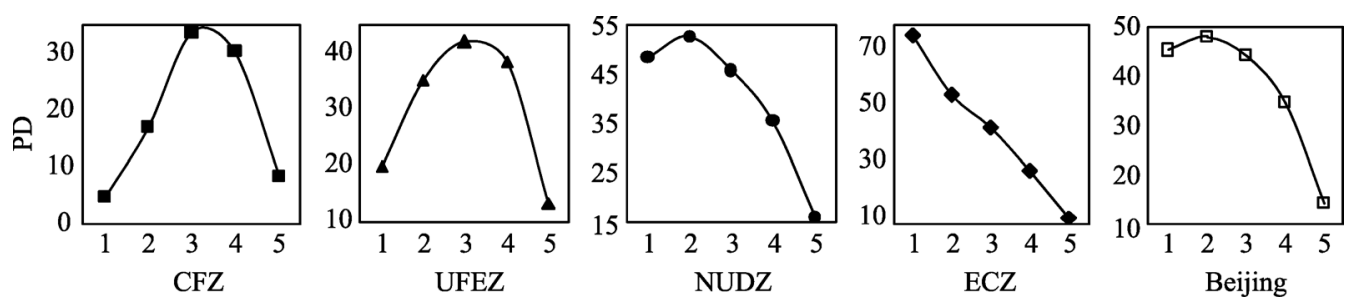

Figure 8 The PD values of different impervious surface's types in four function zones and Beijing in 2015

\section{Discussion}

\subsection{Expansion of impervious surface and contribution of function zones}

The period 1991-2015 is a typical representation of the developed stage of urbanization in 
Beijing. The area and magnitude of impervious surface increased dramatically during this period. Besides, the spatial pattern of impervious surface developed as a single core mode with the impervious surface area expanding from CFZ to NUDZ gradually. These results have been confirmed by many studies (e.g., Wang, 2014; Li et al., 2015; Wang et al., 2015). However, most previous studies primarily focused on the spatio-temporal changes of impervious surface in major zones without considering the differences and heterogeneity among the function zones in Beijing (Kuang et al., 2014).

The distribution of impervious surface among function zones was getting more equal over time. The function zones played different roles in the development of urban impervious surface in Beijing metropolitan region, because of their differences in functions, areas and landscapes. CFZ was the traditional aggregation zone of impervious surface in Beijing and had the highest average ISP values among four zones. However, it only accounts for $8.5 \%$ of the area of Beijing metropolitan region and developed earlier than other zones (Qiao et al., 2013; Wang, 2014). Thus, the area and magnitude of impervious surface in this zone spanned a relatively small range. CFZ is the second main source of the rise of impervious surface in Beijing. UFEZ has the highest CI values among the four zones, and its average ISP values were also much higher than those in Beijing metropolitan region. As a result, UFEZ played the most important role in the growth of impervious surface in Beijing. NUDZ is a major driver for Beijing's future development (State Council Letter [2005] No.2, 2005), and its CI value increased from negative to positive and multiplied, turning to be one of the main sources of the rise of impervious surface. With the economic development and population growth, UFEZ and NUDZ will be the critical zones in the future developments of Beijing metropolitan region. They are the important areas for industrial development and population evacuation (Qiao and Tian, 2014). Therefore, more attention should be paid to these two zones to keep the balance of eco-environments and economic development in the future. Although the impervious surface in ECZ developed rapidly, the average ISP values of this zone were much less than those in Beijing due to its high elevation and dense vegetation. Thus, ECZ had a constant negative contribution, and the CI values also showed a decreasing trend. ECZ plays an important role in protecting water resource and environment in Beijing, and serves as the ecological barrier (Li et al., 2015). However, the absolute values of area of impervious surface in this zone showed an increasing trend and the natural resources were damaged continually, such as the reduction of forests, water areas and wetlands. In the future developments, more attention should be paid to this zone so as to ensure a friendly ecological function in Beijing.

\subsection{Advantages and ecological implications of impervious surface's landscape metrics based on "soft" classifications}

The landscape metrics are more sensitive to the changes of impervious surface derived from the "soft" classifications than the "hard" classifications. The differences of CONTAG values calculated from the "soft" classifications among four zones were greater than those calculated from the "hard" classifications, especially for NUDZ and ECZ (Table 4). Because the small patches are preserved in the results of impervious surface derived from the "soft" classifications, and the local features of landscape can be better reflected by these "soft" 
results (Zou et al., 2014).

Table 4 The comparisons of CONTAG values calculated from the "hard" classifications and "soft" classifications for different function zones in 2015

\begin{tabular}{ccccc}
\hline Classification method & CFZ & UFEZ & NUDZ & ECZ \\
\hline "soft" classifications & 36.90 & 24.17 & 16.03 & 19.96 \\
"hard" classifications & 44.84 & 30.12 & 23.23 & 28.17 \\
\hline
\end{tabular}

The pixel with its ISP retrieved from the "soft" classifications greater than $50 \%$ was regarded as the impervious surface pixel of the "hard" classifications

At the landscape level, the CONTAG values in CFZ were the highest in the four function zones, indicating the spatial aggregation of impervious surface in this zone was the strongest. Besides, the CONTAG values in CFZ decreased at first and then increased. As the key area in the development of Beijing, UFEZ played an important role in the rise of impervious surface. There were two main ways for the growth of impervious surface in this zone. One was increasing the patches of impervious surface outwards, and the other was enhancing the connectedness among patches (Qiao and Tian, 2014). Thus, the spatial connectedness of impervious surface in this zone also decreased at first and then increased. NUDZ and ECZ were the major driver and ecological barrier for the developments of Beijing, respectively. The only way for the growth of impervious surface in these zones was enhancing the connectedness among landscape patches (Kuang et al., 2009). The CONTAG values in the two zones both showed a decreasing trend over time. At the class level, as the ISP rose, the values of LSI and PD both in CFZ and UFEZ firstly increased and then decreased. It means the landscape structure and shape of impervious surface became increasingly fragmented and irregular at first, and then became less fragmented and less complex. In NUDZ and ECZ, the values of LSI and PD showed a downtrend, indicating the fragmentation and complexity of impervious surface landscape attenuated gradually as the ISP rose.

The spatial pattern of impervious surface is one of the important factors influencing the urban ecosystem services, water and thermal environment (May et al., 1997; Xian, 2007; Liu et al., 2013; Zhang et al., 2015). It has close relations with the water pollution and urban heat island (UHI). The rapid growth of impervious surface may aggravate the deterioration of water quality and UHI effects (Yuan and Bauer, 2007; Zhang et al., 2009; Zou et al., 2014). In addition, the high-density impervious surface had the most compact configuration, leading to a greater impact on the urban eco-environment than other types of impervious surface. Therefore, more attention should be paid to the growth and spatial pattern of high-density impervious surface to minimize its increase rate and aggregation.

\section{Conclusions}

The subpixel impervious surface results in Beijing metropolitan region within a time series (1991, 2001, 2005, 2011 and 2015) were derived by means of classification and regression tree (CART) model and change detection models. The spatio-temporal dynamics of impervious surface $(1991,2001,2011$ and 2015) in different function zones and districts were analyzed based on the method of standard deviation ellipse, Lorenz curve, contribution index (CI) and landscape metrics. The following conclusions can be obtained from this study. 
The landscape metrics are more sensitive to the changes of impervious surface derived from the "soft" classifications than the "hard" classifications. This lays a foundation for examining the impact of impervious surface's distribution on eco-environments, and may also offer new insights for exploring the quantitative relationships between impervious surface and ecological factors.

The total area of impervious surface in Beijing grew rapidly during 1991-2015, increasing by about $144.18 \%$. The deflection angle of major axis of standard deviation ellipse decreased from $47.15^{\circ}$ to $38.82^{\circ}$, indicating the major development axis in Beijing moved from northeast-southwest to north-south gradually. Moreover, the heterogeneity of impervious surface's distribution among Beijing's 16 districts decreased, but the CI values and landscape metrics in four function zones differed greatly. The urban function extended zone (UFEZ) had the highest CI values and contributed the most to the growth of urban impervious surface. Its lowest CI value was 1.79 that is still much higher than the highest CI values in other function zones. The core function zone (CFZ), the traditional aggregation zone of impervious surface, had the highest CONTAG values. However, it contributed less than UFEZ due to its small area. The CI value of the new urban developed zone (NUDZ) increased from negative to positive and multiplied, becoming an important contributor to the urban impervious surface. The ecological conservation zone (ECZ) had a constant negative contribution, and the CI value decreased continually. In addition, the landscape metrics and centroids of impervious surface in different density classes differed greatly. The LSI and PD values of high-density impervious surface were the lowest among the five types of impervious surface. It means the high-density impervious surface had the most compact configuration, leading to a greater impact on urban eco-environment than other types of impervious surface in Beijing. The development pattern of high-density impervious surface should be planned and controlled reasonably to minimize its increase rate and aggregation. Furthermore, more attention should be paid to the coordinated development between regional construction and ecological protection to achieve sustainable development in Beijing.

\section{References}

Brun S E, Band L E, 2000. Simulating runoff behavior in an urbanizing watershed. Computers Environment \& Urban Systems, 24(1): 5-22.

Central Committee of the Communist Party of China, the State Council. The National New-type Urbanization Plan (2014-2020). Beijing: Xinhua News Agency, 2014. (in Chinese)

Chester L Arnold Jr, James Gibbons C, 1996. Impervious surface coverage: The emergence of a key environmental indicator. Journal of the American Planning Association, 62(2): 243-258.

Cui Y, Liu J, Qin Y et al., 2015. The impact of urban sprawl on heat island intensity in Beijing. Chinese Journal of Ecology, 34(12): 3485-3493. (in Chinese)

David W S Wong, 1999. Several fundamentals in implementing spatial statistics in GIS: Using centrographic measures as examples. Geographic Information Sciences, 5(2): 163-174.

Fu P, Weng Q, 2016. A time series analysis of urbanization induced land use and land cover change and its impact on land surface temperature with Landsat imagery. Remote Sensing of Environment, 175(4): 205-214.

Gao Z, Zhang L, Li X et al., 2010. Detection and analysis of urban land use changes through multi-temporal impervious surface mapping. Journal of Remote Sensing, 14(3): 593-606. (in Chinese)

Gillies R R, Box J B, Symanzik J et al., 2003. Effects of urbanization on the aquatic fauna of the Line Creek Watershed, Atlanta: A satellite perspective. Remote Sensing of Environment, 86(3): 411-422. 
Hao P, Niu Z, Zhan Y et al., 2015. Spatiotemporal changes of urban impervious surface area and land surface temperature in Beijing from 1990 to 2014. Giscience \& Remote Sensing, 53(1): 1-22.

Huang J, Zhao X, Tang L et al., 2012. Analysis on spatiotemporal changes of urban thermal landscape pattern in the context of urbanization: A case study of Xiamen city. Acta Ecologica Sinica, 32(2): 622-631. (in Chinese)

Kuang W, Chi W, Lu D et al., 2014. A comparative analysis of megacity expansions in China and the U.S.: Patterns, rates and driving forces. Landscape \& Urban Planning, 133(132): 121-135.

Kuang W, Liu J, Lu D, 2011. Pattern of impervious surface change and its effect on water environment in the Beijing-Tianjin-Tangshan metropolitan area. Acta Geographica Sinica, 66(11): 1486-1496. (in Chinese)

Kuang W, Shao Q, Liu J et al., 2009. Spatio-temporal patterns and driving forces of urban expansion in Beijing central city since 1932. Journal of Geo-information Science, 11(4): 428-435. (in Chinese)

Lauren M S, Mark V J, 2010. Spatial statistics in ArcGIS. In: Fischer M M, Getis A (eds.). Handbook of Applied Spatial Analysis: Software Tool, Methods and Applications. Berlin: Springer.

Li X, Gong P, Liang L, 2015. A 30-year (1984-2013) record of annual urban dynamics of Beijing city derived from Landsat data. Remote Sensing of Environment, 166(1): 78-90.

Li Z, Wei Z, Liu Y et al., 2015. Research on Chinese central city impervious surface area growth pattern in recent 20 years: Take Nanchang as a case. Scienta Geographica Sinica, 35(11): 1444-1451. (in Chinese)

Liu T, Yang X, 2015. Monitoring land changes in an urban area using satellite imagery, GIS and landscape metrics. Applied Geography, 56: 42-54.

Liu Y, Wu W, Wen X et al., 2013. Urban process and its eco-environmental impact in Shanxi-Shaanxi-Inner Mongolia energy area. Geographical Research, 32(11): 2009-2020. (in Chinese)

May C W, Horner R R, Karr J R et al., 1997. Effects of urbanization on small streams in the puget sound lowland ecoregion. Watershed Protection Techniques, 1997(4): 79-90.

Nie Q, 2013. Fractal investigation of urban impervious surfaces and its thermal environment effect in Shanghai city [D]. Shanghai: East China Normal University. (in Chinese)

Peng J, 2008. Characteristics analysis of land-atmosphere energy transfer and turbulence over urban and suburban underlying surfaces in Nanjing winter [D]. Nanjing: Nanjing University of Information Science \& Technology. (in Chinese)

Peng J, Liu Y, Shen H et al., 2016a. Using impervious surfaces to detect urban expansion in Beijing of China in 2000s. Chinese Geographical Science, 26(2): 229-243.

Peng J, Wang Y, Zhang Y et al., 2010. Evaluating the effectiveness of landscape metrics in quantifying spatial patterns. Ecological Indicators, 10(2): 217-223.

Peng J, Xie P, Liu Y et al., 2016b. Urban thermal environment dynamics and associated landscape pattern factors: A case study in the Beijing metropolitan region. Remote Sensing of Environment, 173: 145-155.

Qiu J, Sang F, Gao Z, 2011. RS estimating and analysis of urban impervious surface percentage and land surface temperature. Science of Surveying and Mapping, 36(4): 211-213. (in Chinese)

Qiao Z, Tian G, 2014. Spatiotemporal diversity and regionalization of the urban thermal environment in Beijing. Journal of Remote Sensing, 18(3): 715-734. (in Chinese)

Qiao Z, Tian G, Xiao L, 2013. Diurnal and seasonal impacts of urbanization on the urban thermal environment: A case study of Beijing using MODIS data. ISPRS Journal of Photogrammetry \& Remote Sensing, 85(2): 93-101.

State Council Letter [2005] No.2, 2005. Beijing City Master Plan (2004-2020). (in Chinese)

Song Y, 2014. The study of the relationship between impervious surface changes and urban heat island effect in Dianchi Lake Basin based on Landsat data. Kunming: Yunnan Normal University. (in Chinese)

Sun S, 2013. Observation and simulation study on distribution characteristics of radiation and energy balance over Nanjing in summer [D]. Nanjing: Nanjing University of Information Science \& Technology. (in Chinese)

Wang J, Li C, Hu L et al., 2015. Seasonal land cover dynamics in Beijing derived from Landsat 8 data using a spatio-temporal contextual approach. Remote Sensing, 7(1): 865-881.

Wang J, Su G, Kuang W et al., 2014. Spatial and temporal patterns analysis of impervious surface in megacity: A case study of Beijing. Bulletin of Surveying and Mapping, 2014(4): 90-94. (in Chinese) 
Weng Q, 2001. Modeling urban growth effects on surface runoff with the integration of remote sensing and GIS. Environmental Management, 28(6): 737-748.

Xian G, 2007. Analysis of impacts of urban land use and land cover on air quality in the Las Vegas region using remote sensing information and ground observations. International Journal of Remote Sensing, 28(24): $5427-5445$.

Xiao R, Ouyang Z, Cai Y et al., 2007. Urban landscape pattern study based on sub-pixel estimation of impervious surface. Acta Ecologica Sinca, 27(8): 3189-3197. (in Chinese)

Xie M, Wang Y, Li G, 2009. Spatial variation of impervious surface area and vegetation cover based on sub-pixel model in Shenzhen. Resources Science, 31(2): 257-264. (in Chinese)

Yang J, Wang J, Zhang Z, 2012. Inter-provincial discrepancy and abatement target achievement in carbon emissions: A study on carbon Lorenz curve. Acta Scientiae Circumstantiae, 32(8): 2016-2023. (in Chinese)

Yang L, Huang C, Homer C G et al., 2003. An approach for mapping large-area impervious surfaces: Synergistic use of Landsat-7 ETM+ and high spatial resolution imagery. Canadian Journal of Remote Sensing, 29(2): 230-240.

Yuan F, Bauer M E, 2007. Comparison of impervious surface area and normalized difference vegetation index as indicators of surface urban heat island effects in Landsat imagery. Remote Sensing of Environment, 106(3): 375-386.

Zhang D, Guo H, Sun Z, 2012. Estimating surface characteristic parameters in the megacities and the research on their effects towards the urban heat environment. Remote Sensing Technology and Application, 27(1): 51-57. (in Chinese)

Zhang J, Feng Z, Yang Y, 2007. Lorenz curve and its application in the research of spatio-temporal pattern of cultivated land, grain and population in China. Journal of Arid Land Resources and Environment, 21(11): 63-67. (in Chinese)

Zhang L, Gao Z, Liao M et al., 2010. Estimating urban impervious surface percentage with multi-source remote sensing data. Geomatics and Information Science of Wuhan University, 35(10): 1212-1216. (in Chinese)

Zhang Y, Balzter H, Zou C et al., 2015. Characterizing bi-temporal patterns of land surface temperature using landscape metrics based on sub-pixel classifications from Landsat TM/ETM ${ }^{+}$. International Journal of Applied Earth Observation \& Geoinformation, 42: 87-96.

Zhang Y, Odeh I O A, Han C, 2009. Bi-temporal characterization of land surface temperature in relation to impervious surface area, NDVI and NDBI, using a sub-pixel image analysis. International Journal of Applied Earth Observations and Geoinformation, 11(4): 256-264.

Zhou W, Huang G, Cadenasso M L, 2011. Does spatial configuration matter? Understanding the effects of land cover pattern on land surface temperature in urban landscapes. Landscape \& Urban Planning, 102(1): 54-63.

Zou C, Zhang Y, Huang H, 2014. Impacts of impervious surface area and landscape metrics on urban heat environment in Fuzhou city, China. Journal of Geo-information Science, 16(3): 490-498. (in Chinese) 